\title{
MODELO ECONOMÉTRICO DE LOS GASTOS OPERATIVOS DE LA BANCA EN EL ECUADOR: PERIODO 2012 - 2019
}

\section{ECONOMETRIC MODEL OF BANKING OPERATING EXPENSES IN ECUADOR: PERIOD 2012 - 2019}

Eduardo Ron Amores, Mgtr.

https://orcid.org/0000-0003-1483-2994

Magíster en Administración y Dirección de Empresas (Ecuador). Docente de la Universidad ECOTEC, Samborondón, Ecuador. eron@ecotec.edu.ec

Luisa León Vega, Mgtr. https://orcid.org/0000-0002-2253-3890 Magíster en Administración de Empresas (Ecuador). Docente de la Universidad ECOTEC, Samborondón, Ecuador. luleonv@ecotec.edu.ec

Fidel Márquez Sánchez, Ph.D. https://orcid.org/0000-0003-1856-4464 Doctor en Ciencias Económicas (Cuba). Prorrector de la Universidad ECOTEC, Samborondón, Ecuador. fmarquez@ecotec.edu.ec Viviana León León, Mgtr. https://orcid.org/0000-0002-4830-0227 Magíster en Finanzas (Ecuador). Alumni de la Universidad ECOTEC, Samborondón, Ecuador. vileon@mgs.ecotec.edu.ec

\section{ARTÍCULO DE INVESTIGACIÓN}

Recibido: 18 de marzo de 2021

Aceptado: 19 de mayo de 2021

\section{RESUMEN}

Este análisis permite medir el desempeño relativo en cuanto a los expendios administrativos con el que se maneja el sector bancario y poder de esta forma establecer parámetros de comparación de estos. Se basó en un estudio de los gastos que están en propensión a los activos de acuerdo con los recursos que cuentan. Mediante el análisis de regresión de mínimos cuadrados ordinarios, se obtuvo una medida común para cada unidad bancaria. 
Palabras claves: gastos operativos, expendios, banca, modelo econométrico

\section{ABSTRACT}

This analysis makes it possible to measure the relative performance in terms of administrative expenditures with which the banking sector is managed and thus be able to establish comparison parameters for these. It was based on a study of the expenses that are in propensity for the assets according to the resources they have. By means of the regression analysis of ordinary least squares, a common measure was obtained for each banking unit.

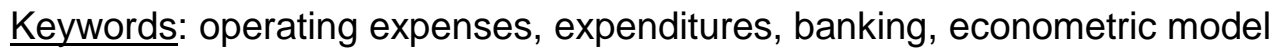

\section{INTRODUCCIÓN}

La banca moderna cumple diferentes funciones dentro de la economía nacional, la composición de sus activos refleja el variado uso de sus recursos (Márquez-Sánchez et al., 2013), destinados a operaciones que le generan una rentabilidad gracias a la cual subió en un marco de creciente competencia. Podríamos afirmar que de la existencia misma de este sector depende la estructura cuantitativa y cualitativa de sus activos. Mantener dicha estructura en niveles óptimos es una de las tareas de mayor importancia para sus administradores (Serrano, 2015).

Los problemas que venían perturbando al sector real de la economía ecuatoriana a fines del siglo pasado y al inicio del actual (Carriel Fuentes et al, 2020), tales como volatilidad de las tasas de interés y del tipo de cambio, acompañados de un proceso inflacionario que amenazaba con desbordar el control por parte de la autoridad monetaria, terminaron minando la estabilidad del sector financiero que entró en crisis, generalizándose la desconfianza de los agentes económicos en el sistema, razón por la que se aceleró la decisión del gobierno de anunciar la adopción del dólar de los Estados Unidos de América como unidad monetaria nacional.

Sin embargo, la dolarización, no resuelve los problemas si no va acompañada de reformas que apoyen el proceso. Es así, que el sistema bancario de nuestro país sufrió una serie de inconveniente, en sus bases estructurales por el cambio al sistema de dolarización, esto a su vez repercutió en los costos de operación de los bancos comerciales y por ende en su desempeño.

El objetivo principal es analizar cuál ha sido el comportamiento de los costos operativos de la banca ecuatoriana en el periodo 2012 - 2019 y qué variables inciden en este comportamiento. 
Los objetivos específicos son:

- Formular un modelo econométrico para los costos operativos de los bancos comerciales del país.

- Realizar la estimación de los coeficientes de regresión a través de intervalos con un nivel de confianza del $99 \%$.

- Hacer las pruebas correspondientes que determinan la validez del modelo.

- Las hipótesis principales fueron:

- Una de las variables fundamentales que incide en los costos administrativos de los Bancos son los activos totales.

- El número de oficinas que tiene los bancos inciden de manera significativa en los costos operativos, estos incluyen agencias y matrices.

- Hay diferencias significativas en los resultados según las diferentes regiones del país.

\section{REVISIÓN TEÓRICA}

\section{Fundamentos teóricos}

El sistema bancario ecuatoriano en el periodo 2012 - 2019 tuvo algunas variantes en cuanto a la conformación de los bancos establecidos en el país, de allí se tuvo que, para el periodo 2012 - 2019; entre bancos que abrieron y entidades bancarias que cerraron existieron 29 bancos (Ver anexo 1). De ahí se tuvo que en el año 2012 constaron 26 bancos; en el año 2013 totalizaron 24 , reduciéndose a 23 entidades financieras bancarias para el año 2014, luego se redujo para el año 2015 a 22 entidades bancarias, en cuanto para el año 2016 permanecieron 23 bancos, y a partir del año 2017 que comprende el periodo 2017 - 2019 se cuantificaron 24 entidades bancarias que tuvieron una importante recuperación.

Si bien en el 2017 se obtuvo total de activos los mismos que se mantuvieron en niveles aceptables por USD 38.975 millones pocos han notado el hecho de que se moderara la tendencia creciente que se había venido registrando tiempo atrás.

El monto de los activos registrados en el sistema bancario privado de Ecuador aumentó cuatro veces en la última década, al pasar de USD 27.875 millones, en el 2012, a USD 44.582 millones en el 2019. Los ahorros se concentran, principalmente, en los cuatro bancos más grandes: Pichincha, Pacífico, Guayaquil y Produbanco. El resto de los activos están distribuidos en 29 entidades bancarias que han existido y finalizado cerrando en diferentes periodos. 
Con relación al crédito, en los últimos años los directivos de los bancos se han replanteado su manera de colocar créditos, por lo que han priorizado poner los recursos en activos productivos de alta rentabilidad (Souto Anido et al, 2021), tanto por sus tasas de interés, que son fijadas por el Banco Central del Ecuador, y por los costos operativos (Diario El Comercio, 2015).

Esto ha hecho, por ejemplo, que se entregue menos créditos de bajo monto para pequeños o medianos productores. Lo que se conoce como microcrédito. La liquidez en el sistema financiera actualmente es mayor, la participación del Banco del IESS (BIESS) en el segmento de vivienda (hipotecarios) y los cambios en el esquema de garantías para casas y autos también son factores que han impulsado a los bancos a cambiar sus políticas de entrega de créditos. A continuación, se presenta los últimos tres años en lo referente a los activos y gastos operacionales de las entidades bancarias y su comportamiento de los 24 bancos que existieron en el país para el periodo 2017 al 2019.

\section{Tabla 1}

\section{Activos y Gastos el Sistema Bancario}

\begin{tabular}{|c|c|c|c|c|c|c|}
\hline \multicolumn{7}{|c|}{ Periodo 2017 - 2019} \\
\hline \multirow{2}{*}{ Bancos } & \multicolumn{2}{|c|}{2017} & \multicolumn{2}{|c|}{2018} & \multicolumn{2}{|c|}{2019} \\
\hline & Activos & Gastos & Activos & Gastos & Activos & Gastos \\
\hline BP Guayaquil & TOTAL ACTIVOS & GASTOS & TOTAL ACTIVOS & GASTOS & TOTAL ACTIVOS & GASTOS \\
\hline BP Pacifico & $4.023 .542,09$ & $376.763,79$ & $4.331 .622,84$ & $414.903,88$ & $5.077 .367,68$ & $456.060,22$ \\
\hline BP Pichincha & $5.451 .933,88$ & $489.430,64$ & $5.533 .587,88$ & $542.699,64$ & $6.082 .137,15$ & $629.368,44$ \\
\hline BP Produbanco & $10.615 .390,88$ & $1.077 .749,19$ & $10.664 .470,22$ & $1.125 .069,01$ & $11.437 .419,48$ & $1.218 .653,69$ \\
\hline BP Austro & $4.271 .783,49$ & $313.619,12$ & $4.768 .269,67$ & $372.286,03$ & $5.171 .926,88$ & $452.136,72$ \\
\hline BP Bolivariano & $1.692 .870,79$ & $163.089,16$ & $1.709 .918,25$ & $172.454,25$ & $1.822 .317,56$ & $182.007,25$ \\
\hline BP Citibank & $3.114 .918,93$ & $196.603,72$ & $3.348 .264,29$ & $215.517,98$ & $3.597 .958,95$ & $256.553,57$ \\
\hline BP General Rumiñahui & $1.674 .489,52$ & $304.676,08$ & $2.171 .397,71$ & $337.582,03$ & $2.445 .773,59$ & $411.049,72$ \\
\hline BP Internacional & $829.859,26$ & $74.788,36$ & $851.804,99$ & $87.602,62$ & $967.161,22$ & $96.975,46$ \\
\hline BP Machala & $446.942,85$ & $35.960,70$ & $481.526,73$ & $39.594,86$ & $523.205,78$ & $45.495,84$ \\
\hline BP Promerica & - & - & - & - & - & - \\
\hline BP Amazonas & $326.280,80$ & $40.040,99$ & $318.530,84$ & $36.076,76$ & $396.221,27$ & $37.342,67$ \\
\hline BP Cofiec & - & - & - & - & - & - \\
\hline BP BancoDesarrollo & $101.845,06$ & $21.686,21$ & $106.082,08$ & $22.323,57$ & $110.180,84$ & $21.713,79$ \\
\hline BP Comercial De Manabi & $165.431,22$ & $17.861,20$ & $202.984,26$ & $21.097,47$ & $216.602,20$ & $25.984,43$ \\
\hline BP Litoral & $57.167,05$ & $5.842,40$ & $51.514,25$ & $6.136,06$ & $55.644,13$ & $6.239,79$ \\
\hline BP Loja & $3.558 .412,08$ & $216.585,31$ & $3.544 .477,38$ & $235.868,78$ & $3.792 .356,29$ & $263.901,71$ \\
\hline BP Solidario & $698.383,71$ & $54.437,27$ & $767.733,14$ & $62.515,32$ & $807.867,29$ & $68.115,54$ \\
\hline BP Sudamericano & - & - & - & - & - & - \\
\hline BP Territorial & - & - & - & - & - & - \\
\hline BP Unibanco & - & - & - & - & - & - \\
\hline BP Coopnacional & $37.496,83$ & $5.079,83$ & $37.502,99$ & $5.080,25$ & $42.201,63$ & $5.266,16$ \\
\hline BP Procredit & $720.162,20$ & $138.943,89$ & $781.940,78$ & $139.466,73$ & $815.068,55$ & $143.056,51$ \\
\hline BP Capital & $185.998,08$ & $11.776,81$ & $188.483,98$ & $14.363,28$ & $186.620,66$ & $11.545,08$ \\
\hline BP Finca & $42.386,25$ & $12.325,28$ & $15.037,69$ & $7.213,92$ & $20.155,24$ & $4.039,77$ \\
\hline BP Delbank & $61.300,76$ & $11.875,27$ & $77.548,58$ & $14.731,29$ & $84.700,19$ & $18.256,13$ \\
\hline BP D-Miro S.A. & $31.544,94$ & $6.906,63$ & $32.908,44$ & $7.225,06$ & $32.183,91$ & $7.383,01$ \\
\hline BP Diners & $642.798,01$ & $50.488,21$ & $757.496,44$ & $44.682,03$ & $634.942,05$ & $40.249,25$ \\
\hline BP VisionFund Ecuador & $168.202,84$ & $23.032,85$ & $169.168,77$ & $24.485,35$ & $179.436,32$ & $28.199,57$ \\
\hline Total Activos & $38.919 .141,55$ & $3.649 .562,90$ & $40.912 .272,21$ & $3.948 .976,17$ & $44.499 .448,84$ & $4.429 .594,32$ \\
\hline
\end{tabular}

Fuente: Estado de situación remitidos por las entidades años 2017-2019. Elaboración: Dirección Nacional de Estudios y Estadísticas /Dirección de Estadísticas del Banco Central. 


\section{REVISION DE LA LITERATURA}

\section{Publicaciones revisadas}

Se toma como referencias dos publicaciones realizadas previamente:

\section{Evolución de los ingresos por servicios financieros de la banca privada ecuatoriana para el período 2013-2017}

Los autores Arias Ronquillo, Ibarra Zuleta, Tulcán Pastas, \& Pastás Gutiérrez (2018) exponen lo siguiente

En la actualidad aparte de los tradicionales servicios financieros de aperturas de cuentas, depósitos y retiros de dinero prestados por los bancos existen otros múltiples servicios cuyo proceso de prestación está siendo ampliado a través de una mejora de su base tecnológica para ofrecer al usuario una mayor agilidad, comodidad y seguridad de ejecución. La pregunta en este punto es si se conoce el comportamiento de la prestación de los servicios financieros en el país y su impacto para el sector de la banca privada, con el objetivo de proyectar hacia dónde se dirigen las nuevas tendencias en la prestación de los citados servicios financieros en el país. Para resolver esta y otras preguntas es necesario analizar, entre múltiples aspectos el comportamiento histórico del factor de ingresos monetarios obtenidos por uno de sus principales oferentes, como es la banca privada nacional.

\section{Educación financiera y factores determinantes: Evidencias desde Ecuador}

Los autores Peñarreta Quezada, García Tinizaray, \& Armas Herrera (2019) expresan lo siguiente; Se analiza la educación financiera de los clientes de la banca ecuatoriana en función de las dimensiones de conocimiento, comportamiento, y aptitud (Márquez-Sánchez et al., 2018). Mediante una encuesta dirigida a 744 personas y a través del uso de técnicas de análisis multivariante, se determinó que solo la tercera parte de la población encuestada tiene educación financiera. Las variables nivel de educación, provincia, género y cargas familiares son las más influyentes en el estudio para explicar el nivel de educación financiera.

\section{Conceptualización:}

\section{Superintendencia de Bancos}

La Superintendencia de Bancos es un organismo técnico de derecho público, encargada de efectuar la vigilancia, auditoría, intervención, control y supervisión de las actividades financieras que ejercen las entidades públicas y privadas del sistema financiero ecuatoriano, atendeindo el interés general (Asamblea Nacional, act. 2019). 
Sus principales funciones son:

a) Vigilar que las instituciones financieras cumplan con la legislación aplicada al sistema financiero y los principios de equidad, transparencia, justicia y sanas prácticas.

b) Defender los derechos y generar confianza de los ciudadanos en el sistema financiero, atendiendo sus peticiones y orientándolos sobre las condiciones del mismo.

c) Promover la transparencia de la información.

d) Contribuir a que la mayor cantidad de usuarios tengan acceso al sistema financiero

\section{Banca privada}

La banca privada consiste en una gestión altamente profesionalizada y global del patrimonio de un cliente. Se trata de satisfacer las necesidades de inversión, planificación patrimonial, financiera y fiscal de personas o grupos familiares con un elevado patrimonio (Superintendencia de Bancos, 2019).

La banca privada se dedica por tanto a tareas de asesoramiento financiero y gestión patrimonial. Para que un servicio de banca privada sea como tal, tiene que producirse una relación banco-cliente que destaque por ofrecer un servicio personalizado.

\section{Sistema financiero ecuatoriano}

Un sistema financiero es el conjunto de instituciones que tiene como objetivo canalizar el ahorro de las personas. Esta canalización de recursos permite el desarrollo de la actividad económica (producir y consumir) haciendo que los fondos lleguen desde las personas que tienen recursos monetarios excedentes hacia las personas que necesitan estos recursos (Noboa Salazar et al., 2021). Los intermediarios financieros crediticios se encargan de captar depósitos del público y, por otro, prestarlo a los demandantes de recursos (Superintendencia de Bancos, 2019).

\section{Análisis del sistema bancario ecuatoriano}

En el Ecuador de las dos últimas décadas, sus bancos competían permanentemente con mucha publicidad, por sobre los niveles de eficiencia, para captar la mayor cantidad de clientes, los cuales no siempre se sentían bien atendidos. En términos generales, nuestros bancos fueron típicos de una economía periférica en la que destacaban la concentración del crédito y su poca eficacia para captar ahorro (Sorhegui-Ortega et al., 2021), dichos recursos no se encausaron hacia actividades productivas y siempre se pensó que si un banco tenía problemas el Estado debía estar listo para salvarlo (García Osorio, 2016). 
En contrario y para su beneficio, se podía decir que la banca ecuatoriana gozó de muy buen nivel tecnológico, en comparación con la infraestructura bancaria de otros países de la región. Nuestras instituciones se preocuparon de ofrecer buena imagen. Incluso algunas comenzaron costosos procesos de reingeniería.

Esa tecnología no siempre le sirvió al cliente, que lejos de ser bien atendido con créditos oportunamente entregados y a bajo costo, más bien sirvió de pretexto para encarecer los servicios hacia los cuales era atraído, artificiosamente, a atreves de rifas y sorteos. En todo caso, como la característica primordial de un banco consiste en guardar fondos ajenos en forma de depósitos, operaciones denominadas de pasivo. Por la salvaguarda de estos fondos, los bancos cobran una serie de comisiones, que también se aplican a los distintos servicios que los bancos modernos ofrecen a sus clientes en un marco cada vez más competitivo: tarjetas de crédito, banco telefónico, entre otros. Sin embargo, puesto que el banco puede disponer del ahorro del depositante, remunera a este último mediante el pago de un interés. Podemos distinguir varios tipos de depósitos (Tenesaca, Villanueva, Malo, \& Higuerey, 2017).

En primer lugar, los depósitos pueden materializarse en las denominadas cuentas corrientes: el cliente cede al banco unas determinadas cantidades para que éste las guarde, pudiendo disponer de ellas en cualquier momento. Tiempo atrás, hasta adquirir carácter histórico, este tipo de depósitos no estaban remunerados, pero la creciente competencia entre bancos ha hecho que esta tendencia haya cambiado de forma drástica en todos los países.

En segundo lugar, los bancos ofrecen cuentas de ahorro, que también son depósitos a la vista, es decir, que se puede disponer de ellos en cualquier momento. Los depósitos y reintegros se realizan y quedan registrados a través de una cartilla de ahorro, que tiene carácter de documento financiero. La disponibilidad de este tipo de depósitos es menor que la de las cuentas corrientes puesto que obligan a recurrir a la entidad bancaria para disponer de los fondos, mientras que las cuentas corrientes permiten la disposición de fondos mediante la utilización de cheques y tarjetas de crédito.

En tercer lugar, hay que mencionar las denominadas cuentas a plazo fijo, en las que no existe una libre disposición de fondos, sino que éstos se recuperan a la fecha de vencimiento, aunque, en la práctica, se puede disponer de estos fondos antes de la fecha prefijada, pero con una penalización (la remuneración del fondo es menor que en el caso de esperar a la fecha de vencimiento). 
En cuarto lugar, dentro de los distintos tipos de depósitos, los depósitos de ahorro vinculado son cuentas remuneradas relacionadas con operaciones bancarias de activo (es el caso de una cuenta vivienda: las cantidades depositadas deben utilizarse para un fin concreto, como es el caso de la adquisición de vivienda).

Los bancos, con estos fondos depositados, conceden préstamos y créditos a otros clientes, cobrando a cambio de estas operaciones (denominadas de activo) otros tipos de interés, estos préstamos pueden ser personales, hipotecarios o comerciales. La diferencia entre los intereses cobrados y los intereses pagados constituye la principal fuente de ingresos de los bancos. Además, los bancos también ofrecen servicios de cambio de divisas, permitiendo que sus clientes compren unidades monetarias de otros países (Aguilera, 2015).

En la actualidad, el sistema bancario ecuatoriano conserva dos características del sistema utilizado anteriormente.

a) Los pasivos monetarios del sistema bancario exceden las reservas; esta característica permitió, en parte, el proceso de industrialización occidental y sigue siendo un aspecto muy importante del actual crecimiento económico. Sin embargo, la excesiva creación de dinero puede acarrear un crecimiento de la inflación.

b) Los pasivos de los bancos (depósitos y dinero prestado) son más líquidos, es decir, se pueden convertir con mayor facilidad que el oro en dinero en efectivo que los activos (préstamos a terceros e inversiones) que aparecen en su balance. Uno de los principales cometidos de los bancos centrales es regular el sector de la banca comercial para minimizar la posibilidad de que un banco entre en esta situación y pueda arrastrar tras él a todo el resto del sistema bancario.

Se han obtenido los datos del sistema bancario ecuatoriano y más concretamente de los bancos privados, de la web de la Superintendencia de Bancos, según se detalla en tabla Nº 2 . Se confecciona un panel completo de 29 entidades bancarias comprendidas para un periodo de 8 años y que está comprendido entre 2012-2019. 


\section{Tabla 2}

Bancos Privados. Periodo 2012 - 2019

\begin{tabular}{|ll}
\hline No. & \multicolumn{1}{c}{ Bancos } \\
\hline 1 & BP Guayaquil \\
2 & BP Pacifico \\
3 & BP Pichincha \\
4 & BP Produbanco \\
5 & BP Austro \\
6 & BP Bolivariano \\
7 & BP Citibank \\
8 & BP General Ruminahui \\
9 & BP Internacional \\
10 & BP Machala \\
11 & BP Promerica \\
12 & BP Amazonas \\
13 & BP Cofiec \\
14 & BP BancoDesarrollo \\
15 & BP Comercial De Manabi \\
16 & BP Litoral \\
17 & BP Loja \\
18 & BP Solidario \\
19 & BP Sudamericano \\
20 & BP Territorial \\
21 & BP Unibanco \\
22 & BP Coopnacional \\
23 & BP Procredit \\
24 & BP Capital \\
25 & BP Finca \\
26 & BP Delbank \\
27 & BP D-Miro S.A. \\
28 & BP Diners \\
29 & BP VisionFund Ecuador \\
\hline & \\
\hline
\end{tabular}

Fuente: Superintendencia de Bancos. Elaboración: Los autores

\section{MATERIALES Y MÉTODOS}

\section{Localización de la investigación}

La presente investigación fue realizada con información estadística de la Superintendencia de Bancos de la República del Ecuador.

\section{Periodo de investigación}

El periodo de investigación fue desde el periodo 2012 - 2019

\section{Universo}

Para el estudio, se consideraron todos los Bancos privados que ofrecen sus servicios al sector público como privado. 


\section{Diseño de la investigación}

Esta investigación se clasifica como correlacional, porque se espera encontrar la correlación por medio de los estudios econométricos y estadísticos (Oramas Santos et al, 2018), entre la variable independiente activos y la variable dependiente gastos. Intrínsecamente los métodos científicos para el desarrollo eficiente de esta investigación (Hernández rojas et al., 2021), se utilizará el método inductivo, así se recolectará y analizará los datos generados por las variables dependiente e independiente, para clasificarlos y estudiarlos (Jimber del Río et al., 2020), esto es con el fin de contar con información óptima y aprovechar la utilidad del método deductivo.

Se desarrolló una investigación cuantitativa (Hernández, Fernández, \& Baptista, 2014) utilizando el análisis de regresión de mínimos cuadrados ordinarios, a fin de tener una medida común para sus costos administrativos en función del volumen de activos y él número de oficinas que tienen los bancos.

\section{Tipo de investigación}

El tipo de investigación fue exploratoria y descriptiva, con enfoque cuantitativo.

\section{Técnicas de análisis de datos}

Los datos de todas las variables fueron tomados de las tablas estadísticas registradas en la Superintendencia de Bancos aplicando el método de regresión múltiple donde las variables exógenas serán los activos totales (act) de los bancos y los gastos totales (gtos), de este modo se creará un modelo econométrico con datos en series de tiempo, y consecutivamente se efectuará las correspondientes pruebas para corregir la posible auto correlación, endogeneidad, heterosedasticidad y multicolinealidad del modelo, ajustando de forma correcta el modelo para que no haya errores de explicación ecuacional por medio de la prueba de examen de residuos (Souto Anido et al., 2019).

\section{Fuentes de información}

Entre las fuentes de información se encuentra el estado de situación financiera remitido por las entidades al organismo de control.

\section{Manejo de la investigación}

La investigación se realizó previamente bajo un protocolo de trabajo para la recolección de información de los indicadores operacionales bancarios correspondientes al periodo 2012 - 2019 y para la presentación de resultados por medio de tablas estadísticas con información de activos de bancos privados. 


\section{Formulación del modelo}

Para el análisis de los gastos operativos la variable explicativa escogida fue el total de activos de los bancos que actualmente operan en el país. Se detalla a continuación en la siguiente tabla:

\section{Tabla 3}

Matriz de Impacto de gastos operacionales

\begin{tabular}{|ccc|}
\hline \multirow{3}{*}{ MATRIZ DE IMPACTO DE GASTOS OPERACIONALES } \\
\hline \multicolumn{3}{c|}{ (en miles dólares) } \\
\cline { 2 - 3 } & ACTIVOS & GASTOS \\
\hline \multirow{2}{*}{ ANNOS } & act & gtos \\
\hline & $27.875 .396,0$ & $2.634 .778,0$ \\
$\mathbf{2 0 1 2}$ & $30.738 .406,7$ & $2.754 .167,2$ \\
$\mathbf{2 0 1 3}$ & $33.619 .120,7$ & $3.023 .250,7$ \\
$\mathbf{2 0 1 4}$ & $30.864 .079,1$ & $3.248 .900,2$ \\
$\mathbf{2 0 1 5}$ & $35.599 .114,7$ & $3.265 .959,0$ \\
$\mathbf{2 0 1 7}$ & $38.974 .957,3$ & $3.658 .936,8$ \\
$\mathbf{2 0 1 8}$ & $40.983 .994,8$ & $3.961 .255,0$ \\
$\mathbf{2 0 1 9}$ & $44.582 .648,0$ & $4.444 .410,0$ \\
\hline
\end{tabular}

Fuente: Superintendencia de Bancos - Estados de situación remitidos por las entidades, (2012 - 2019)

Los instrumentos para recopilar los datos obtenidos se basarán en las bases obtenidas de la Superintendencia de Bancos formato excel y por medio del programa econométrico Stata, con lo cual se obtendrá el modelo econométrico, empleando asi las pruebas respectivas y poder conseguir un modelo estadísticamente significativo y de alta confiabilidad. A partir de los datos obtenidos, la metodología de este estudio se divide en 4 fases se detallan a continuación:

1. Habiendo definido el fenómeno económico a estudiar, su espacialidad (activos y gastos por entidad bancaria); y temporalidad (8 años), en el ciclo de especificación se realizó el anàlisis de la información obtenida en la base de datos de la Superintendencia de Bancos, la cual fue llevada a cabo por medio de la clasificación de las variables dependiente e independientes o explicativas, de esta manera fue posible establecer los tipos de datos de cada variable, la escala de medida, y los niveles indicados en la Tabla 3, su naturaleza matemática. Luego de obtener estas definiciones se efectuó un análisis descriptivo de la variable endógena. 
2. La base de datos formada para la estimación del modelo activos - gastos totales en bancos del Ecuador, fue establecida para una muestra de 8 años (periodo 2012 - 2019); que proporcionan información a nivel nacional, donde los activos totales (act) es asumida como variable independiente, mientras que gastos totales (gtos) es la variable dependiente con las que se explicará el comportamiento de los bancos a nivel nacional.

3. Una vez obtenidos los datos, éstos fueron organizados y estructurados en una tabla matriz Excel, acto seguido, los datos fueron importados al software econométrico Stata para su posterior análisis.

4. En la fase estimación se efectuó el cálculo de los parámetros del modelo por medio de un análisis estadístico de regresión lineal y partiendo de la técnica de mínimos cuadrados ordinarios (MCO), luego de conseguir los valores de los coeficientes para cada variable explicativa, se procedió a seleccionar aquellas variables cuyas betas fueran significativos con una probabilidad del 10\% mediante la utilización de la regresión lineal múltiple.

La metodología utilizada se sustenta en la metodología tradicional o tradicional de la econometría (Vergara-Romero et al, 2020), está sujeta a los lineamientos que se presentan en el siguiente esquema:

\section{Estadística descriptiva}

\section{Variable exógena}

Tabla 4.

Estadísticas Variable Explicada

\begin{tabular}{|r|ccccc|}
\hline summarize act & & & & & \\
Variable & Obs & Mean & Std. Dev. & Min & Max \\
\hline act & 8 & $3.54 e+07$ & 5737322 & $2.79 e+07$ & $4.46 e+07$ \\
\hline
\end{tabular}

Fuente: Tabla 3, Stata / SE

\section{Correlación variable exógena}

\section{Matriz de correlación de variables exógenas}

A partir de la tabla 4, el valor esperado o valor promedio de act corresponde a USD 35.404'714,7 mil, el valor de la desviación estándar que muestra el grado de dispersión de los datos de la muestra con respecto a su valor promedio corresponde a 5.737.322 
El rango de valores posibles abarca desde el valor mínimo USD 27.875’396,0 mil hasta el máximo valor USD 44.582'648,0 mil, sugiriendo que valores bajos de act corresponden a los activos de los bancos durante el periodo $2012-2019$.

Tabla 5.

Correlación de Variables

\begin{tabular}{|r|rr|}
\hline pwcorr act gtos & \\
& act & gtos \\
\hline act & 1.0000 & \\
gtos & 0.9606 & 1.0000 \\
\hline
\end{tabular}

Fuente: Tabla 3

La tabla anterior explica cómo se encuentran relacionadas cada una de las variables entre si al interior del modelo. Las variables cuyos valores están cercanos a cero, por ejemplo, el coeficiente de correlación entre gtos respecto de act es de 0.9606 indicando que la correlación es alta, esto es, ambas variables son linealmente dependientes (si influye una en la otra). Es asi que, el signo que acompaña el coeficiente es positivo sugiriendo que la proyección de la regresión lineal va a tender a crecer conjuntamente una variable con relación de la otra (variables directamente proporcionales).

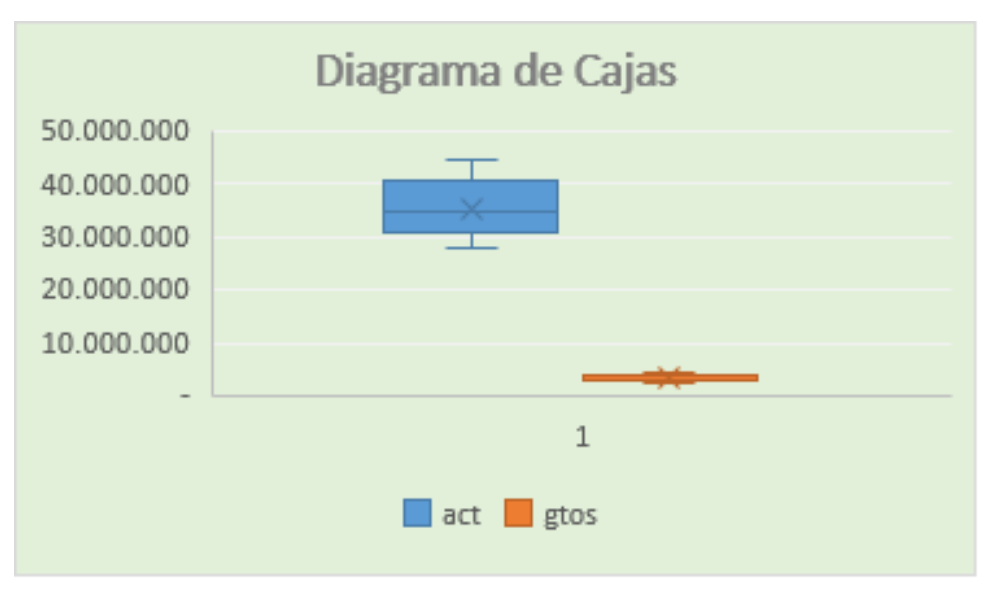

Figura 1. Diagrama de cajas. Fuente: Tabla 5

Como se visualiza en la Figura 1, con la representación gráfica del diagrama de cajas aquí se obtiene la distribución de cada una de las variables en términos de su mediana, percentiles, valores extremos y su dispersión. Con lo cual permite comparar entre sí cada una de las variables explicativas. 


\section{RESULTADOS}

El modelo inicial presentado, que involucra todas las variables consideradas en la metodología es el siguiente:

act $=5199381+8.952495$ gtos

Tabla 6

Modelo Inicial de Activos (act)

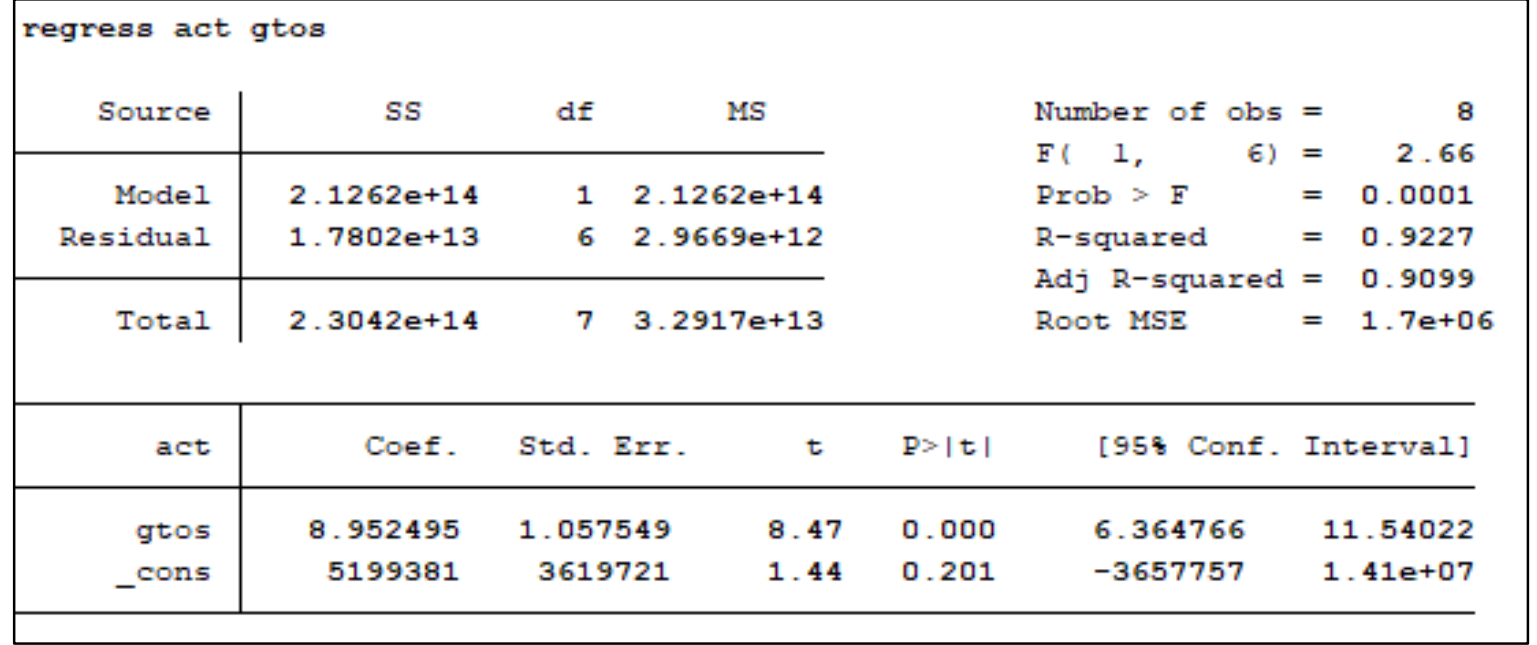

Fuente: Tablas 3, 4 y 5

Como puede observarse en la salida anterior, todos los coeficientes son estadísticamente significativos, pues los $p$ valores son inferiores al 10\%. De modo que al aplicar la regresión paso a paso Stepwise con una probabilidad del $10 \%$ se obtuvieron todas las variables significativas: gtos con un $\mathrm{p}$ valor de 0.0 , el coeficiente posee un $\mathrm{p}$ valor superior a la significancia del $5 \%$, por lo que es aceptada la hipótesis nula, por ende, este sería el modelo definitivo para act.

En cuanto a la naturaleza de los datos están expresados como indicadores, es decir que los coeficientes que acompañan a cada una de las variables explicativas representan el cambio marginal, es decir, que si una de las variables aumenta en una unidad y las demás toman valores de 0.0001 , el act en promedio cambia en el valor de beta unidades de dicha variable considerados en la muestra. Realizando el analisis para $\mathrm{R}^{2}$, se tiene que es de 0.9227 , por lo que la simplicidad del ajuste, que intenta resumir es que: las variables exógenas explican el comportamiento de act, y este es del $92.27 \%$. 
Con respecto a la significancia individual y global del modelo, dada una significancia del 10\%, los valores críticos de t corresponden a [1.44, 8.47], en consecuencia, los estadísticos de prueba t calculados para los Beta; obteniendo esto se concluye que los hallazgos para estos coeficientes son de gran significancia. Teniendo el significado global, dado que el p valor que acompaña el estadístico $F$ es significativo ( $p$ valor de 0.0001 ); se logra conocer que no hay evidencia estadística para aceptar la hipótesis nula, así de este modo y en conjunto con los estimadores de las pendientes (betas) del modelo tienden a ser significativos.

En cuanto a la verificación de los supuestos del modelo clásico de regresión lineal (MCRL) se ejecutó la prueba estadística que diferencia la hipótesis a partir del test de autocorrelación de orden uno o Durbin Watson se hizo uso de la prueba test de autocorrelación, con el mismo se obtuvo un valor $\mathrm{DW}=2.711903$, y dados un $\mathrm{dl}=2$ y un $\mathrm{du}=8$ se comprueba que el modelo cae en la zona de no autocorrelación de primer orden, se demuestra a continuación:

$\mathbf{H}_{0}$ : No existe autocorrelación , $\mathbf{H}_{1}$ : Existe autocorrelación

Tabla 7 .

Prueba Test Durbin-Watson

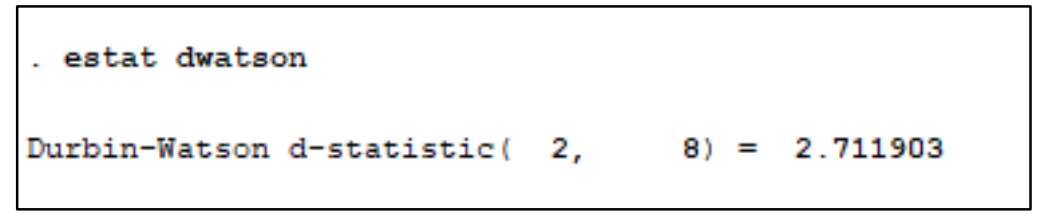

Fuente: Tabla 6

Con esto se logró obtener un valor superior a la significancia del $5 \%$ por lo que es aceptada la hipótesis nula y se afirma que no hay cambio estructural.

A un nivel muy elemental, por supuesto, dicha variación no tiene realmente ningún misterio, ya que los bancos varían mucho en cuanto a su tamaño, y es más bien de esperarse que los bancos más "grandes" tengan también gastos operacionales más altos por el sólo hecho de ser de mayor cobertura administrativa. La tarea será traducir esta noción intuitiva en un concepto operativo, y para esto se debe tratar de expresar el tamaño de un banco en término de alguna variable numérica.

Cabe indicar que, en un estudio de este tipo las variables que afectan los costos administrativos son múltiples, sin embargo, para mantener este análisis aplicable a todos los bancos se escogió una variable fundamental. Esa variable fue el volumen promedio de activos de los bancos en el periodo 2012 - 2019 y se procedió a realizar una aproximación. 
Previo a utilizar el análisis, fue necesario hacer ciertas modificaciones a los datos originales. Primero se deflactaron los datos, la razón de hacer esto es para evitar introducir sesgo a los resultados pues los niveles de la crisis país para el año 2020 en el Ecuador es muy elevada. Luego se calculó el activo promedio de los bancos para cada año. La razón de usar el activo promedio está en evitar problemas de comparabilidad.

\section{CONCLUSIONES}

Luego de culminar el desarrollo y análisis del tema "Modelo Econométrico de los Gastos Operativos de la Banca en el Ecuador, período 2012 - 2019", se puede concluir lo siguiente:

La crisis bancaria que experimentó el país hace algunos años, ha influido negativamente, lo cual se evidencia en el bajo crecimiento económico.

Además, hay que aumentar que en la vida económica moderna la banca se ha constituido en un papel fundamental e importante para el desarrollo del país; ya que, una de las funciones más importantes que posee la banca es el de otorgar créditos; mediante el crédito se desenvuelve los capitales y se realizan hechos fundamentales, como la producción, circulación y consumo. Se acepta por lo tanto al crédito como el eje de los adelantos del mundo económico.

En términos generales, los bancos ecuatorianos fueron típicos de una economía periférica en la que destacaban la concentración del crédito. Las instituciones bancarias se preocuparon en ofrecer buena imagen, incluso algunas comenzaron con costosos procesos de reingeniería y tecnología de punta; pero esa tecnología no siempre le sirvió al cliente que lejos de ser bien atendido con créditos oportunamente entregados, se le cobra elevados costos, por los gastos operativos que tienen los bancos.

Uno de los factores determinante en los costos administrativos de la banca en el Ecuador es el monto de activos de los bancos, Debe tenerse en mente que estas variables no son estrictamente independientes ya que, para poder incrementar sus activos, un banco deberá captar más depósitos, mientras que su captación depende en buena medida del número de agencias que controla.

\section{REFERENCIAS BIBLIOGRÁFICAS}

Aguilera, F. (2015). El impacto de la crisis financiera y económica internacional en la banca del Ecuador. Quito: Universidad Andina Simón Bolívar / Corporación Editora Nacional. 
Arias Ronquillo, C. J., Ibarra Zuleta, F. O., Tulcán Pastas, A. L., \& Pastás Gutiérrez, E. R. (2018). Evolución de los ingresos por servicios financieros por servicios financieros de la banca privada ecuatoriana para el período 2013-2017. Espacios, 39(47), 3-13. Obtenido de http://www.revistaespacios.com/a18v39n47/a18v39n47p03.pdf

Asamblea Nacional. (act. 2019). Código Orgánico Monetario y Financiero. Quito: Registro Oficial.

Carriel Fuentes, O.C., Márquez-Sánchez, F., Pinzon Benalcazar, Y. \& Sorhegui-Ortega, R. (2020). Sector informal: Tributación y Desarrollo local. Revista Espacios, 41(15), 21. https://www.revistaespacios.com/a20v41n15/a20v41n15p21.pdf

Diario El Comercio. (25 de enero de 2015). Conozca cómo se ha comportado su banco en la última década. Diario El Comercio. Recuperado el 16 de febrero de 2020, de https://www.elcomercio.com/datos/comportamiento-banco-ultima-decada.html

García Osorio, N. (2016). Evolución de la Banca Privada despues de la crisis financiera en el Ecuador 2000 - 2010. Sangolquí: Universidad de las Fuerzas Armadas ESPE.

Hernández, R., Fernández, C., \& Baptista, P. (2014). Metodología de la Investigación (Sexta ed.). México: McGraw-Hill / Interamericana de Editores S.A. de C.V.

Hernández-Rojas, R. D., Jimber del Río, J. A., Ibañez Fernández, A., \& Vergara-Romero, A. (2021). The cultural and heritage tourist, SEM analysis: the case of The Citadel of the Catholic King. Heritage Science, 9(1), 1-19.

Jimber del Río, J. A., Hernández-Rojas, R. D., Vergara-Romero, A., \& Dancausa Millán, M. (2020). Loyalty in Heritage Tourism: The Case of Córdoba and Its Four World Heritage Sites. International Journal of Environmental Research and Public Health, 17(23), 8950.

Márquez-Sánchez, F., Macías Gradín, I., Manosalvas del Pezo, J., \& Sorhegui-Ortega, R. (2018). La reforma tributaria y su impacto en la liquidez fiscal y empresarial en Ecuador, periodo 2010-2016. Espacios, 39(8), 3

Márquez-Sánchez, F., Sorhegui-Ortega, R., \& Bayón Sosa, M. (2013). Pensamiento Económico Universal Preclásico.

Noboa Salazar, J., Vergara-Romero, A., Sorhegui-Ortega, R., \& Garnica-Jarrin, L. (2021). Repensando el desarrollo sostenible en el territorio. RES NON VERBA REVISTA CIENTÍFICA, 11(1), 19-33. https://doi.org/10.21855/resnonverba.v11i1.500 
Oramas Santos, O., Ortiz Torres, M., Souto Anido, L, \& Márquez-Sánchez, F. (2018). Herramientas Matemáticas como Vía para el Diseño de Sistemas de Estimulación Laboral. GECONTEC: Revista Internacional de Gestión del Conocimiento y la Tecnología, 6(2), 61-74. Recuperado de https://upo.es/revistas/index.php/gecontec/article/view/3735

Peñaherrera Quzada, M., García Tinizaray, D., \& Armas Herrera, R. (2019). Educación financiera y factores determinantes: Evidencias desde Ecuador. Espacios, 40(7), 11-25. Obtenido de http://www.revistaespacios.com/a19v40n07/a19v40n07p11.pdf

Serrano, A. (2015). La nueva economía en la nueva Constitución del Ecuador. Quito: Centro de Estudios Fiscales / Servicio de Rentas Internas del Ecuador.

Sorhegui-Ortega, R.; Vergara-Romero, A.; Garnica-Jarrin, L. (2021). Economía post-crecimiento: una visión de múltiples perspectivas teóricas. Estudios del Desarrollo Social: Cuba y América Latina, 9(2), 209-223.

Souto Anido, L., Márquez-Sánchez, F., Marrero Ancizar, Y. (2019). Procedimiento para la selección del personal basado en la Teoría de los Subconjuntos Borrosos y Operadores OWA. Revista espacios, 40(34), 9. Recuperado de http://www.revistaespacios.com/a19v40n34/19403409.html

Souto-Anido, L., Vergara-Romero, A., Marrero-Anciza, Y., \& Márquez-Sánchez, F. (2021). Incidencia de la Gestión de los Recursos Humanos en los resultados Organizacionales: ¿mito o realidad? ( Impact of Human Resources Management on Organizational Results: Myth or Reality?). GECONTEC: Revista Internacional De Gestión Del Conocimiento Y La Tecnología, 8(1), 1-23. Recuperado de https://upo.es/revistas/index.php/gecontec/article/view/5410

Superintendencia de Bancos. (2012 - 2019). Portal estadístico / Bancos. Recuperado el 1 de marzo de 2020, de Estados de situación remitidos por las entidades / Sistemas de $\begin{array}{lll}\text { Administración de Balances } & \text { (S.A.B.): }\end{array}$ http://estadisticas.superbancos.gob.ec/portalestadistico/portalestudios/?page_id=415

Superintendencia de Bancos. (2019). Educación Financiera. Recuperado el 16 de mayo de 2020, de Portal del usuario financiero: https://estadisticas.superbancos.gob.ec/portalestadistico/efinanciera/?page_id=38 
Tenesaca, K. X., Villanueva, J., Malo, Z., \& Higuerey, Á. (2017). Sistema bancario del Ecuador: una aproximación a sus indicadores de estabilidad y eficiencia. Publicando, 4(13), 255273.

Vergara-Romero, A., Olalla Hernández, A., Yturralde, J.M., \& Sorhegui-Ortega, R. (2020). Responsabilidad social corporativa y su impacto en el rendimiento económico de las principales Empresas en Ecuador. Revista ESPACIOS, 41(10). 


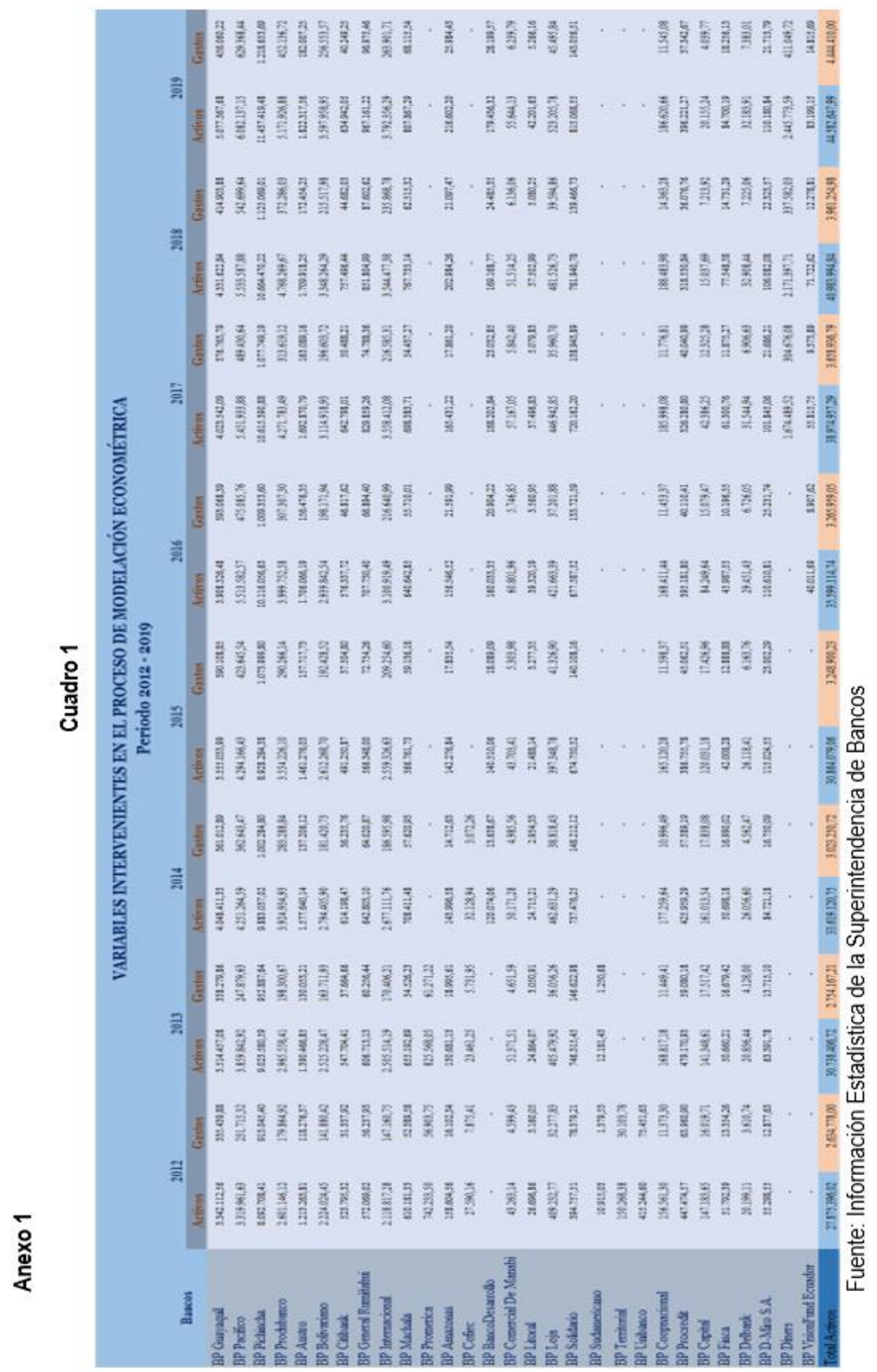

